\title{
Necroptosis: a crucial pathogenic mediator of human disease
}

\author{
Mary E. Choi, ${ }^{1,2}$ David R. Price, ${ }^{2,3}$ Stefan W. Ryter, ${ }^{3}$ and Augustine M. K. Choi ${ }^{2,3}$ \\ 'Division of Nephrology and Hypertension, Joan and Sanford I. Weill Department of Medicine, Weill Cornell Medicine, New \\ York, New York, USA. ${ }^{2}$ New York-Presbyterian Hospital, Weill Cornell Medical Center, New York, New York, USA. ${ }^{3}$ Division of \\ Pulmonary and Critical Care Medicine, Joan and Sanford I. Weill Department of Medicine, Weill Cornell Medicine, \\ New York, New York, USA.
}

Necroptosis is a genetically regulated form of necrotic cell death that has emerged as an important pathway in human disease. The necroptosis pathway is induced by a variety of signals, including death receptor ligands, and regulated by receptor-interacting protein kinases 1 and 3 (RIPK1 and RIPK3) and mixed-lineage kinase domain-like pseudokinase (MLKL), which form a regulatory necrosome complex. RIPK3-mediated phosphorylation of MLKL executes necroptosis. Recent studies, using animal models of tissue injury, have revealed that RIPIK3 and MLKL are key effectors of injury propagation. This Review explores the functional roles of RIPIK3 and MLKL as crucial pathogenic determinants and markers of disease progression and severity in experimental models of human disease, including acute and chronic pulmonary diseases; renal, hepatic, cardiovascular, and neurodegenerative diseases; cancer; and critical illness.

Conflict of interest: AMKC is a cofounder of, is a stockholder of, and serves on the Scientific Advisory Board for Proterris, Inc., which develops therapeutic uses for carbon monoxide. AMKC also has a use patent (US $7,678,390)$ on CO. AMKC served as a consultant for Teva Pharmaceuticals in July 2018. The spouse of MEC is a cofounder of, is a shareholder of, and serves on the Scientific Advisory Board of Proterris, Inc.

Copyright: (c) 2019 American Society for Clinical Investigation

Reference information: JCl Insight. 2019;4(15):e128834. https://doi. org/10.1172/jci.insight.128834.

\section{Introduction}

Cell death is a natural endpoint of normal cell physiology that results in irreversible termination of cellular functions, including growth, division, and metabolic homeostasis. Recent discoveries have reshaped our understanding of the complex modes by which eukaryotic cells die during normal growth and development, in response to physiological injury, and during progression of disease. Classical nomenclature has segregated cell death modalities into those regulated by genetic programs and those that are circumstantial but not genetically regulated (1-3). Since its discovery in Caenorhabditis elegans, apoptosis has come to represent the archetypal form of programmed cell death that occurs during development and the predominant form of regulated cell death (RCD) triggered by adverse environmental stimuli. In contrast, lytic cell death, which arises catastrophically in response to external injurious stimuli or adverse physicochemical conditions, was defined as non-RCD and termed "necrosis." These definitions were grounded in distinct morphological and biochemical observations of dying cells $(2,3)$. Apoptosis requires the activation of cysteine proteases and endonucleases without loss of plasma membrane integrity. Morphologically, apoptosis is characterized by cytosolic shrinkage, membrane blebbing, chromatin condensation, and DNA fragmentation (4). In contrast, necrosis is defined by loss of energy charge, cell swelling, and plasma membrane damage, resulting in cytosolic constituents leaking into the extracellular space, which may trigger local inflammation and damage to surrounding tissues (3). These conventional cell death definitions began to blur as mixed modalities of cell death that exhibit features of apoptosis and morphological characteristics that overlap with necrosis (referred to as "aponecrosis") were recognized $(5,6)$.

Further discoveries have elucidated additional cell death pathways, leading to the paradigm-shifting revelation that certain cell death types are morphologically similar to necrosis but can also be regulated by underlying genetic programs $(1,7,8)$. Cell death nomenclature now groups apoptosis with distinct forms of regulated necrosis under the umbrella of RCD and excludes nonregulated necrosis, now classified as accidental cell death (ACD) (1). The forms of RCD are represented by mitochondrial membrane permeability transition-dependent (MPT-dependent) necrosis, parthanatos, ferroptosis, pyroptosis, necroptosis, and other modalities as recently reviewed (refs. 1, 7; see Table 1 and Figure 1).

Necroptosis shares several ACD-associated morphological features, including organelle swelling, plasma membrane rupture, cell lysis, and leakage of intracellular components, which in turn may propagate secondary inflammatory responses (9-11). Similar to nonregulated necrosis, necroptosis represents an inflammatory mode of cell death (12). The necroptosis pathway is regulated by distinct proteins, namely 
Table 1. Comparative morphological and biochemical features of RCD

\begin{tabular}{|c|c|c|c|c|}
\hline Cell death mode & Genetic regulation & Morphological/biochemical features & Key regulatory molecules ${ }^{A}$ & Refs. \\
\hline Apoptosis & $\mathrm{Y}$ & $\begin{array}{c}\text { Membrane blebbing, cell shrinkage, chromatin } \\
\text { condensation, DNA fragmentation, mitochondrial } \\
\text { dysfunction, cytochrome } c \text { release, altered Bcl-2 family } \\
\text { protein expression and activation }\end{array}$ & $\begin{array}{l}\text { (+) Caspase }-8,-9,-3 \text {, and }-7 \text {; Bax; Bad; } \\
\text { Bid; SMAC/DIABLO. (-) BcI-2, BcI-X } \\
\text { IAP1/2, XIAP. }\end{array}$ & $1-4$ \\
\hline Necrosis & $\mathrm{N}$ & $\begin{array}{l}\text { Plasma membrane rupture, cell swelling and lysis, energy } \\
\text { decline, DAMP release }\end{array}$ & None & $1-4$ \\
\hline Necroptosis & Y & $\begin{array}{l}\text { Plasma membrane rupture, cell swelling and lysis, energy } \\
\text { decline, DAMPs release }\end{array}$ & (+) RIPK1, RIPK3, MLKL. (-) Caspase-8. & $7-9$ \\
\hline Ferroptosis & Y & $\begin{array}{l}\mathrm{Fe}^{2+} \text {-dependent membrane lipid oxidation and membrane } \\
\text { damage }\end{array}$ & $(+) \mathrm{Fe}^{2+} \cdot(-) \mathrm{GPX} 4$ & $28-30$ \\
\hline Pyroptosis & Y & $\begin{array}{c}\text { Inflammasome activation membrane rupture, cell } \\
\text { swelling and lysis, pore-induced intracellular traps, DNA } \\
\text { fragmentation, nuclear condensation, DAMP release, } \\
\text { proinflammatory cytokines }\end{array}$ & (+) Caspase- 1 and -7, GSDMD. & \\
\hline Cellular senescence & $\mathrm{N}$ & Irreversible inhibition of the cell cycle & $\begin{array}{l}\text { (+) p53, cyclin-dependent kinase } \\
\text { inhibitor p16. }\end{array}$ & $\begin{array}{c}1,94, \\
142\end{array}$ \\
\hline
\end{tabular}

receptor-interacting protein kinases 1 and 3 (RIPK1 and RIPK3) and downstream substrate pseudokinase mixed-lineage kinase domain-like (MLKL) $(8,9)$.

The necroptosis pathway has been implicated as both an adaptive and pathogenic component of many human pathologies that involve inflammatory processes, including atherosclerosis, cardiac ischemia/reperfusion (I/R) injury, sepsis, inflammatory bowel disease, and neurodegenerative diseases (7-9, 13). Necroptosis has also emerged as an instrument of innate immunity during infectious diseases and is considered a protective mode of cell death that eliminates pathogen-infected cells $(10,14)$. Furthermore, necroptosis activation is an integral component of autoimmune and autoinflammatory diseases $(15,16)$ and may also contribute to the pathogenesis of genetic diseases, such as Duchenne's muscular dystrophy and lysosomal storage disorders $(17,18)$.

Emerging studies indicate that RIPK3 and/or RIPK1 orchestrate key cellular responses in tissue injury. Moreover, as signaling kinases, RIPKs have a potential sphere of influence that extends beyond canonical regulators of the necroptosis pathway and includes functions, such as cell death-independent regulation of proinflammatory pathways $(6,12,19,20)$ and innate immune responses $(21)$. Recent studies have described RIPK3-dependent phenotypes in diverse models of organ injury and disease that may be context dependent or independent of MLKL $(7,8)$. Furthermore, the injury or disease model may dictate the involvement of necrotic cell death and activation of inflammatory pathways. Here, we examine recent findings on the contribution of necroptosis and associated signaling regulators, such as RIPK3, to human diseases involving inflammation or organ injury. The complex role of necroptosis signaling proteins as regulators of cell death, inflammation, and metabolism; mediators of tissue injury; and circulating markers of disease progression and severity will be discussed. Necroptosis may affect the propagation of acute injuries, where cell death is a critical component of pathogenesis, as well as chronic diseases, which may also involve tissue remodeling and immunomodulation.

\section{RCD modes and their relationship to necroptosis}

Regulation of apoptosis is divided into 2 routes: the extrinsic pathway, which is regulated by death receptor-dependent caspase- 8 , and the intrinsic pathway, which is regulated by receptor-independent initiation of mitochondrial dysfunction that in turn leads to cytoplasmic translocation of mitochondrial cytochrome $c$ and activation of caspase-9. Both pathways converge on caspase-3 activation, to initiate apoptosis (1). 

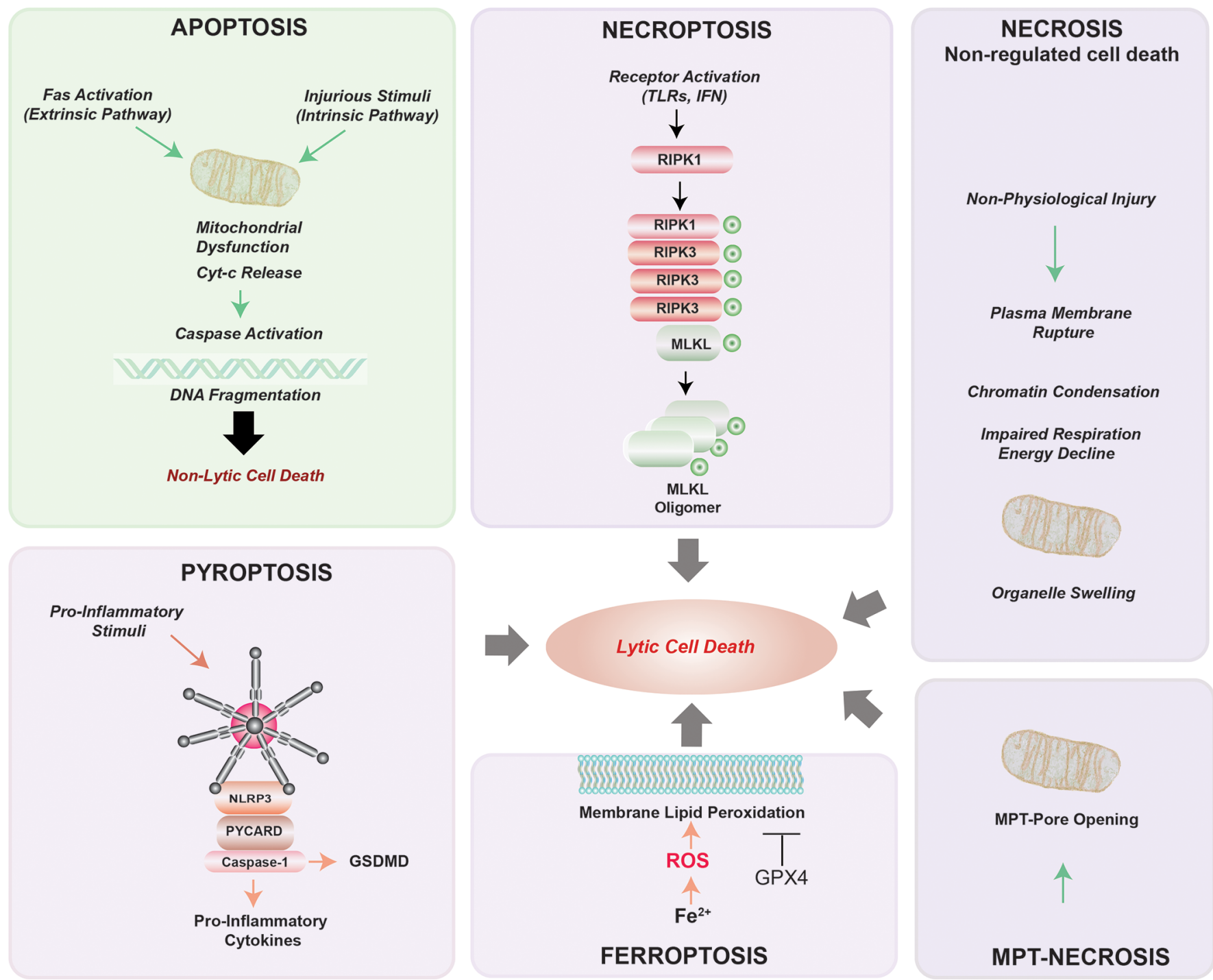

Figure 1. Modes of cell death. Cell death pathways include those that are regulated by genetic programs, which are designated regulated cell death (RCD), and those that are not regulated, which are designated accidental cell death (ACD). Apoptosis is a form of RCD that requires activation of caspases, leading to DNA fragmentation. The initiation of apoptosis may be receptor dependent (extrinsic pathway) or triggered by injurious stimuli (intrinsic pathway), resulting in mitochondrial dysfunction. Apoptosis culminates in nonlytic cell death, which is noninflammatory. Pyroptosis is a form of lytic cell death that occurs in inflammatory cells in response to proinflammatory stimuli. A cardinal feature of pyroptosis is the requirement for inflammasome-dependent caspase-1 activation, which regulates the maturation and secretion of proinflammatory cytokines. Pyroptosis occurs as the result of gasdermin D-regulated (CSDMD-regulated) membranous pore formation and features cytoplasmic swelling and cytosolic content leakage. Necroptosis represents a form of RCD that is activated by RIPK1 and requires RIPK3-dependent phosphorylation of MLKL. MLKL oligomerization results in plasma membrane rupture, leading to a lytic form of cell death associated with release of DAMPs. Ferroptosis is an RCD mode that is distinct from necroptosis. In ferroptosis, iron-dependent lipid peroxidation causes lytic cell death, which can be inhibited by glutathione peroxidase 4 (CPX4). Necrosis is a lytic form of ACD that results in DAMP release and propagation of inflammation. A variant of necrosis that involves mitochondrial permeability transition (MPT) has also been described. DAMPs, damage-associated molecular patterns; MLKL, mixed-lineage kinase domain-like pseudokinase; NLRP3, nucleotide-binding oligomerization domain-, leucine rich repeat-, and pyrin domain-containing protein 3; PYCARD, PYRIN-PAAD-DAPIN domain- and C-terminal caspase-recruitment domain-containing protein; RIPK1, receptor-interacting protein kinase 1; RIPK3, receptor-interacting protein kinase 3.

Cell fate decision (apoptosis vs. necroptosis) is influenced primarily by the availability of caspase- 8 and the cellular or X-linked inhibitors of apoptosis proteins (cIAP1, cIAP2, XIAP) (8). cIAPs govern RIPK1 ubiquitinylation and degradation $(22,23)$, whereas caspase- 8 mediates proteolytic cleavage of RIPK1 and RIPK3 $(24,25)$. Caspase- 8 or cIAP deficiency favors necroptosis by, respectively, removing proteolytic cleavage of RIPK1/RIPK3 or ubiquitinylation of RIPK1 (22-25). Caspase-8 deficiency is embryonic lethal in mice, via upregulation of regulated necrosis; however, RIPK3 and MLKL are dispensable for embryonic development. Inhibition of regulated necrosis and Ripk3 or Mlkl deficiency can rescue embry- 
onic lethality in caspase-8-null mice $(15,26,27)$. In TLR-dependent cell death, RIPK1 participates in regulatory signaling complexes that involve Fas-associated death domain (FADD), caspase- 8 , and caspase- 8 inhibitor $\mathrm{CFLIP}_{\mathrm{L}}$ and are independent of the necroptosis pathway (8).

Ferroptosis is a recently described RCD mode that is distinct from necroptosis and that represents an iron-dependent form of lytic cell death and results from excessive membrane lipid peroxidation, which is under tonic regulation by GPX4 $(11,28-30)$.

Pyroptosis is a form of lytic cell death that occurs primarily in inflammatory cells, such as macrophages, but also in nonimmune cells, such as endothelial cells, and is triggered by bacteria, pathogens, or their endotoxins $(1,11,31)$. Because necroptosis and pyroptosis are lytic forms of cell death, they share similarities, including release of DAMPs and inflammation propagation; however, their underlying molecular regulation and ability to limit local tissue damage are distinct (32). In contrast with unchecked DAMP release from necroptotic cells (33), pathogen-infected cells undergoing pyroptosis maintain membranes after lysis, trapping pathogens in a pore-induced intracellular trap and simultaneously releasing DAMPs and awaiting neutrophil efferocytosis to limit further local inflammation (34).

\section{Molecular mechanisms of necroptosis pathway regulation}

The necroptosis pathway can be initiated during impaired apoptosis by ligand-dependent stimulation of cell surface death receptors, such as Fas, TNF receptor 1 (TNFR1), IFN receptors (IFNRs), TLRs, and intracellular RNA- or DNA-sensing molecules (ref. 35 and Figure 2). RIPK1 was originally identified as a mediator of caspase-independent cell death effectuated by these pathways (36). TNF-dependent stimulation of TNFR1 has 3 functional outcomes that are dependent on regulatory protein assembly. These divergent pathways culminate in stimulation of NF- $\mathrm{kB}$-dependent inflammation, caspase-8-dependent apoptosis, or alternative activation of the necroptosis pathway under conditions of caspase-8 inhibition (8). TNF-driven necroptosis is regulated by RIPK1 and RIPK3, which interact via intrinsic RIP homotypic-interacting motifs (RHIMs) (37, 38). RIPK1 and RIPK3 interaction leads to autophosphorylation, transphosphorylation, and assembly of a "necrosome" complex (39-41). Inhibition of RIPK1 activity with necrostatin-1 can inhibit necroptosis (42), and RIPK3-deficient mice survive to adulthood, implying that RIPK3 is nonessential for development (43), whereas Ripk1-knockout mice die perinatally (44). Kinase-deficient RIPK3 ${ }^{\text {D161N }}$ mice die during embryogenesis by RIPK1-dependent apoptosis, whereas kinase-deficient RIPK1 ${ }^{\mathrm{D} 138 \mathrm{~N}}$ mice are viable $(20,45-47)$.

IFN stimulation of IFNR also triggers RIPK1-RIPK3 necrosome formation and necroptosis in macrophages (48). In contrast, TLR stimulation activates a RIPK1-independent necroptosis pathway via TRIF. In response to TLR4 stimulation by LPS or TLR3 stimulation by dsRNA, TRIF complexes with RIPK3 to activate MLKL- and RIPK1-independent cellular necroptosis (49). Other necroptosis mediators include the DAI. DAI binds cytosolic dsDNA to promote production of type I IFNs (50) and can also interact with RIPK3, via RHIM domains, to activate MLKL independently of RIPK1.

The necrosome complex consists of interacting RIPK1-RIPK3 proteins that assume a higher-order amyloid structure $(51,52)$. RIPK3-mediated MLKL phosphorylation is a key event in necroptosis execution that promotes MLKL oligomerization and subsequent assimilation into the plasma membrane, where it promotes membrane permeabilization and calcium efflux (53). Phosphorylation of the MLKL pseudokinase domain uncovers a latent $\mathrm{N}$-terminal 4-helix bundle (4HB) domain required for cell lytic activity (54). MLKL oligomer binding to plasma membrane phosphatidylinositol phosphates triggers membrane pore opening (55), and MLKL assimilation at the plasma membrane may also recruit ion channels, leading to flux of ions such as $\mathrm{Ca}^{2+}, \mathrm{Na}^{+}$, and $\mathrm{K}^{+}$.

Recent studies have identified novel cellular mechanisms for regulation of necroptosis. MLKL-dependent membrane damage is inhibited by endosomal sorting complexes required for transport (ESCRT-III), a regulator of endosome trafficking (56). Braking of necroptotic cell death by ESCRT-III permits the release of signaling molecules before cell lysis (56). MLKL can also regulate endosome formation. RIPK3-dependent phosphorylation of MLKL results in binding of p-MLKL to ESCRT-III, which promotes the extracellular vesicle formation (57). The extracellular export of p-MLKL in vesicles may represent a mechanism for autoattenuation of necroptosis (57). Furthermore, RIPK3 is subjected to posttranslational modification by $O$-GlcNAc transferase (OGT), which catalyzes $O$-GlcNAcylation of RIPK3 at threonine 467, which impairs necrosome formation. OGT deletion enhances innate immune activation and promotes septic inflammation (58).

\section{Necrotic DAMPs}

MLKL-dependent necroptotic membrane permeabilization subsequently induces release of cellular cyto- 


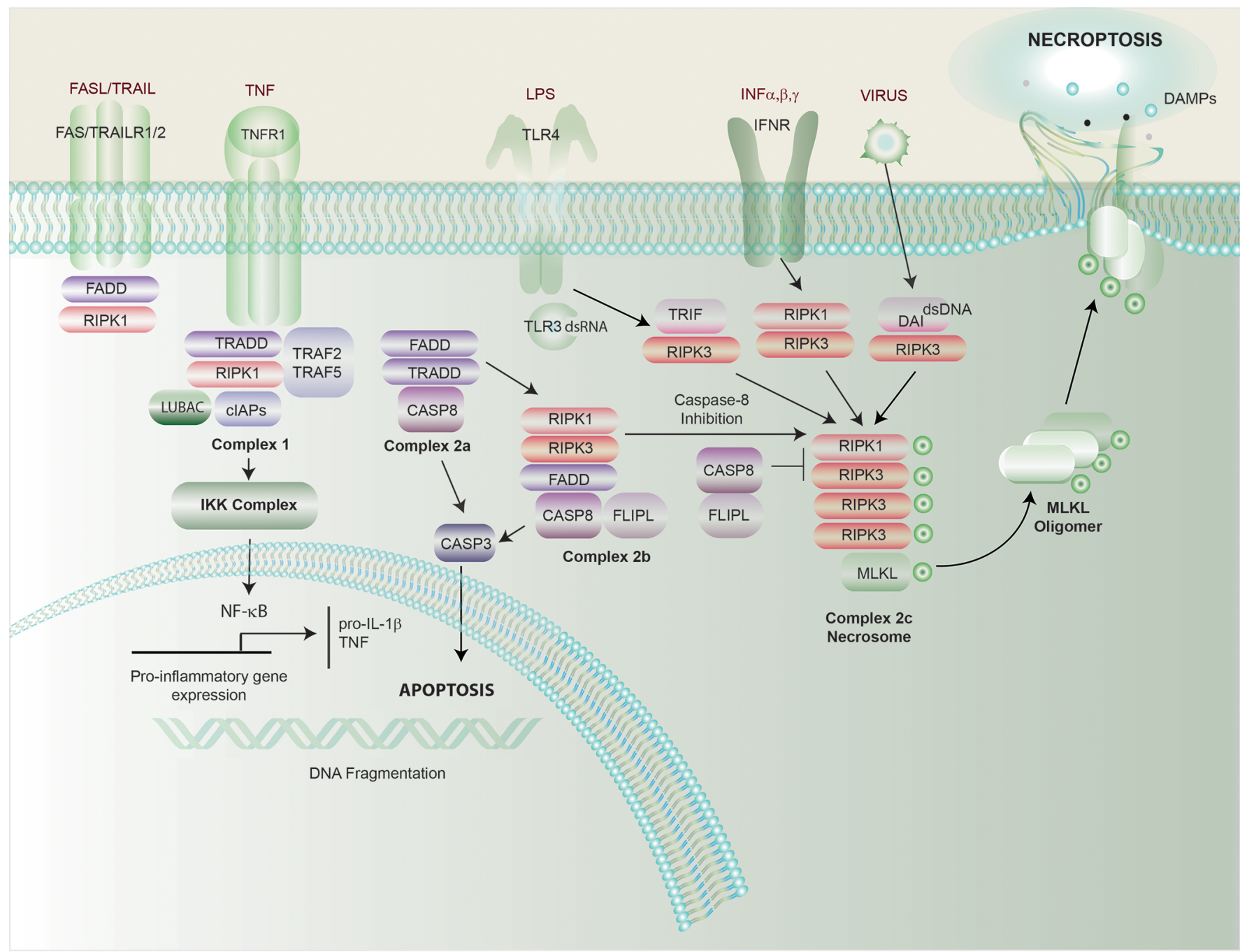

Figure 2. Regulation of necroptosis, apoptosis, and inflammation. Stimulation of TNFR1 by TNF results in differential activation of proinflammatory and proapoptosis pathways. TNFR1 activation results in complex formation between RIPK1 and TNFR1-associated death domain (TRADD) protein and other accessory proteins. Ubiquitinylation of RIPK1 by cellular inhibitor of apoptosis proteins (cIAPs) or linear ubiquitin chain assembly complex (LUBAC) results in recruitment of the inhibitor of $\mathrm{I}-\kappa \mathrm{B}$ kinase (IKK) complex, leading to nuclear translocation and activation of NF- $\mathrm{KB}$. NF- $\mathrm{KB}$ regulates transcriptional activation of genes important for cell survival, proinflammatory cytokines, and apoptosis-related proteins. Alternatively, a second deubiquitinylated complex of RIPK1 associates with Fas-associated death domain (FADD) protein and pro-caspase-8. The resulting caspase- 8 activation leads to activation of the extrinsic apoptosis pathway, and activation of effector caspase-3 and -7 , culminating in apoptosis. Under conditions of caspase-8 depletion or clAP deficiency, stimulation of TNFR1 promotes the necroptosis pathway. Necrosome formation involves RIPK1/RIPK3 interaction and activation of MLKL. Alternative receptor/ligand interactions, such as stimulation of TLR3/TLR4 by dsRNA or LPS or sensing of viral DNA by DNA-dependent activator of IFN-regulatory factors (DAI), leads to the formation of a TRIF-RIPK3 complex or DAI-RIPK3 complex and RIPK1-independent necrosome formation. Phospho-MLKL ( $p$-MLKL) oligomerizes and translocates into the plasma membrane, where it causes membrane rupture and release of DAMPs. TRAF, TNF receptor associated factor; TRAIL, TNF-related apoptosis-inducing ligand; TRIF, Toll/IL-1 receptor domain-containing adapter-inducing IFN- $\beta$.

plasmic material and specific DAMPs that can promote inflammation in distal tissues $(6,59,60)$. DAMP-dependent propagation of systemic inflammation and potential feedback to necrotic cell death together is referred to as "necroinflammation" (59). Several necrosis-associated DAMPs, including high-mobility group box 1 (HMGB1) and ATP, have been identified $(6,10)$. Additionally, proinflammatory cytokines and chemokines may be released into the circulation as the consequence of inflammasome activation and cell lysis by pyroptosis or necroptosis (60). Mitochondrial DNA (mtDNA) may serve as a cellular DAMP following release from the mitochondria under proinflammatory conditions (61). Moreover, mtDNA is measurable in the systemic circulation in inflammatory diseases, such as sepsis (62). The specific role of mtDNA as a necroptosis-related DAMP requires further characterization. 


\section{Regulation of inflammation by necroptosis-associated proteins}

Necroptosis-associated proteins indirectly propagate inflammation by promoting release of necrotic DAMPs via cell lysis. Necroptosis signaling kinases may directly regulate inflammatory pathways (6, 60). RIPK1 can induce cytokine production by acting as a scaffolding protein, independently of its kinase activity and of RIPK3, and participates in regulatory complex formation during TNF/NF- $\mathrm{BB}$ activation, which results in proinflammatory cytokine gene transcription $(46,63,64)$. Recent studies suggest that both RIPK1 and RIPK3 mediate NF- $\mathrm{BB}$ activation in vivo during acute inflammatory responses to LPS (65).

Recent studies have introduced mechanistic roles for RIPK3/MLKL in inflammasome regulation. The nucleotide-binding oligomerization domain- (NOD-), leucine-rich repeat- (LRR-), and pyrin domaincontaining protein-3 (NLRP3) inflammasome governs proinflammatory cytokines' maturation and secretion (66). NLRP3 inflammasome activation involves assembly of adaptor protein PYCARD and procaspase-1, which triggers caspase-1-dependent processing of proinflammatory cytokines (66).

RIPK3-dependent NLRP3 inflammasome regulation in response to TLR4 activation occurs under conditions of inhibition or deficiency of apoptosis components; however, the physiological relevance remains incompletely understood $(8,67)$. In the absence of cIAPs, RIPK3, independent of kinase activity, and caspase- 8 are required for TLR4-mediated NLRP3 inflammasome activation. Both RIPK3 activity and MLKL are required for inflammasome activation under combined deficiencies of cIAPs and caspase-8 (67). RIPK3, but not MLKL, is required for NLRP3 inflammasome activation in cIAP-deficient mice (67). In a model of macrophage activation by LPS stimulation and caspase inhibition, MLKL was required for caspase-1 activation and IL-1 $\beta$ maturation (68). MLKL-dependent inflammasome activation requires MLKL oligomerization, $4 \mathrm{HB}$ domain activation, plasma membrane assimilation, and $\mathrm{K}^{+}$ion efflux, while MLKL-dependent IL-1 $\beta$ secretion is independent of pyroptosis and cell lysis (68). In contrast, inhibition of NLRP3 inflammasome activation did not inhibit MLKL-driven necroptosis, suggesting that MLKL-dependent inflammation may selectively contribute to the pathogenesis of certain diseases (68). Caspase- 8 deficiency promoted RIPK1/ RIPK3-dependent inflammasome activation in response to TLR4 stimulation (69). A RIPK1/RIPK3/ MLKL/caspase-8 axis was implicated in posttranslational regulation of NLRP3 inflammasome assembly in response to TLR3 stimulation with dsRNA (70). A relationship between RIPK3 and the regulation of innate immune responses in the CNS was shown in a model of viral encephalitis (21). RIPK3 promoted inflammatory cell recruitment and viral clearance independently of MLKL and cell death (21). Further studies are needed to unravel the complex relationship between necroptosis and innate immune responses.

\section{Mitochondria and necroptosis}

Mitochondria are important for energy production and integral to the maintenance of cellular metabolic pathways. Although mitochondrial dysfunction has been characterized in inflammatory responses, such as NLRP3 inflammasome activation, the role of these organelles in necroptosis regulation remains incompletely delineated (Figure 2). RIPK3 was proposed to be required for mitochondrial ROS (mtROS) production during necroptosis activation (71), and antioxidants, such as butylated hydroxyanisole (BHA), inhibit TNF-driven necroptosis activation (72). The involvement of mitochondria in necroptosis regulation was challenged by a study that demonstrated that TNF-mediated necroptosis activation is not compromised in cells severely depleted of mitochondria by parkin overexpression and treatment with mitochondrial depolarizing agents (73). This study demonstrated that BHA inhibition of the necroptosis pathway is not specific for mtROS (73).

The mitochondrial molecule phosphatase phosphoglycerate mutase 5 (PGAM5) was initially implicated as a necroptosis regulator via a mechanism involving mobilization of dynamin-related protein 1 (DRP1) to initiate mitochondrial fission (74). Later observations, using genetic deletion strategies, challenged these studies and suggested PGAM5 is dispensable for necroptosis activation by TNF in the presence of caspase inhibition (75-77). Deletion of Drp1 or the mitophagy regulator PTEN-inducible putative kinase 1 (Pink1) did not compromise TNF-induced necroptosis in L929 cells (77). In contrast, PINK1 is required for RIPK3 expression in cigarette smoke-exposed (CS-exposed) epithelial cells (78), suggesting altered mitochondrial function may regulate non-receptor-mediated, stress-induced necroptosis via mitophagy.

We recently observed that RIPK3 may act upstream of mitochondrial dysfunction to regulate tissue injury. In kidney tubular epithelial cells, RIPK3 promotes oxidative stress and mitochondrial dysfunction involving coordinated upregulation of mitochondrial NADPH oxidase 4 (NOX4) and inhibition of mitochondrial complexes I and III (79). RIPK3 was required for increased mitochondrial translocation 
of NOX4 in response to proinflammatory stimuli by a mechanism involving NOX4-RIPK3 interactions, implicating RIPK3 as a stimulator of mtROS production under sepsis conditions (79).

\section{Necroptosis-associated proteins in pulmonary disease}

Recent studies have implicated the necroptosis pathway and its regulatory proteins in pulmonary diseases and related disorders of critical care medicine (80-82). An emerging paradigm is that RIPK3 is a crucial mediator of tissue injury in murine models of acute lung injury (ALI), sepsis-associated organ injury, and chronic lung diseases (Figure 3).

$A L I$. The contribution of necroptosis-associated proteins to ALI has recently been investigated in a mouse model of ventilator-induced lung injury (VILI). In mice subjected to injurious mechanical ventilation (MV) at high tidal volume $(25 \mathrm{~mL} / \mathrm{kg})$, Ripk3 deficiency conferred protection against lung inflammation and injury relative to WT mice. In contrast, MLKL-deficient mice were not resistant to VILI (83), suggesting that RIPK3 may act as a signaling mediator to enhance inflammation and tissue injury, independent of MLKL. In the VILI model, chemical inhibition of the fatty acid $\beta$-oxidation (FAO) pathway with perhexiline and etomoxir aggravated lung injury. Moreover, FAO inhibitor-associated injury in this model was abolished in RIPK3-deficient mice, suggesting a link among mitochondria-dependent FAO, RIPK3 signaling, and tissue injury development (83).

Concomitant RIPK3-dependent necroptosis and RIPK3-dependent inflammasome activation were observed in an LPS-induced ALI model. Moreover, RIPK3-specific inhibitor GSK'872 ameliorated lung injury and reduced inflammatory cell influx and IL-1 $\beta$ and IL-18 production in this model. In THP-1 macrophages subjected to LPS- and ATP-induced NLRP3 inflammasome activation, a novel RIPK3/ NLRP3 complex, leading to caspase-1 activation and IL-1 $\beta$ and IL-18 production, was described. These studies suggest that inflammasome and necroptosis pathways are coregulated in the pathogenesis of lung inflammatory injury (84).

Sepsis/systemic inflammatory response syndrome. The relationship between inflammation and necroptosis was investigated in a mouse model of TNF-induced systemic inflammatory response syndrome (SIRS) (49). Loss of caspase-3, caspase-7, or caspase-1 was not protective in this model. Conversely, Ripk3 deficiency conferred protection against lethal SIRS and reduced circulating DAMPs. Pharmacological application of RIPK1 inhibitor necrostatin-1 and genetic inactivation of RIPK1 activity also provided protection in this model $(49,50)$, indicating that RIPK1- and RIPK3-mediated cellular damage by necroptosis drives mortality during TNF-induced SIRS. In the same model, RIPK1-inactive (RIPK1 $1^{\mathrm{D} 138 \mathrm{~N} / \mathrm{D} 138 \mathrm{~N}}$ ) chimeric mice reconstituted with hematopoietic cells from WT mice were protected from TNF-induced SIRS (85), while mice reconstituted with RIPK1-inactive hematopoietic cells were not protected. These results suggest that the protection from TNF-induced SIRS in RIPK1-inactive mice is mediated by abrogated necroptosis in nonhematopoietic cell lineages (85). Ripk3 deficiency also protected against cecal ligation and punctureinduced (CLP-induced) polymicrobial sepsis, as evidenced by improved survival and reduction of systemic DAMPs and proinflammatory cytokine production (49). Subsequent studies of Ripk3 deficiency in the CLP model have reported conflicting results $(86,87)$. We have observed that RIPK3, but not MLKL, mediates sepsis-induced acute kidney injury (AKI) (79). In a mouse model of cecal slurry-induced neonatal sepsis, inhibition of RIPK1 with necrostatin-1 decreased systemic and pulmonary inflammation, reduced lung injury, and improved survival (88). Similarly, Ripk3 deficiency attenuated serum and lung cytokine production, lung injury, neutrophil infiltration, and lung apoptosis in this model. These data suggest that RIPK3 contributes to a systemic inflammatory response in neonatal sepsis (89).

Chronic pulmonary diseases. Chronic obstructive pulmonary disease (COPD) involves loss of alveolar surface area (emphysema) and airway inflammation (bronchitis) as the consequence of aberrant inflammatory responses to chronic CS exposure. Pulmonary epithelial cells represent a primary target of inhaled CS, although pathological responses in fibroblasts and inflammatory cells may contribute to lung dysfunction (90). We have observed complex integration and regulation of cell death pathways, including evidence of apoptosis, necrosis, and necroptosis in cellular and tissue models of CS-induced lung epithelial cell injury $(78,91-93)$. CS induced extrinsic apoptosis in cultured pulmonary epithelial cells, with dose-dependent transition to necrosis at higher concentrations $(78,91)$. CS-induced epithelial cell senescence has also been characterized (94). Notably, these cell death-associated responses are integrated with dose-dependent regulation of autophagy. Mitochondria represent a primary target of CS-induced cellular injury, as manifested by mitochondrial dysfunction, depolarization, and mtROS production. CS also promoted activation of Drp1-reg- 


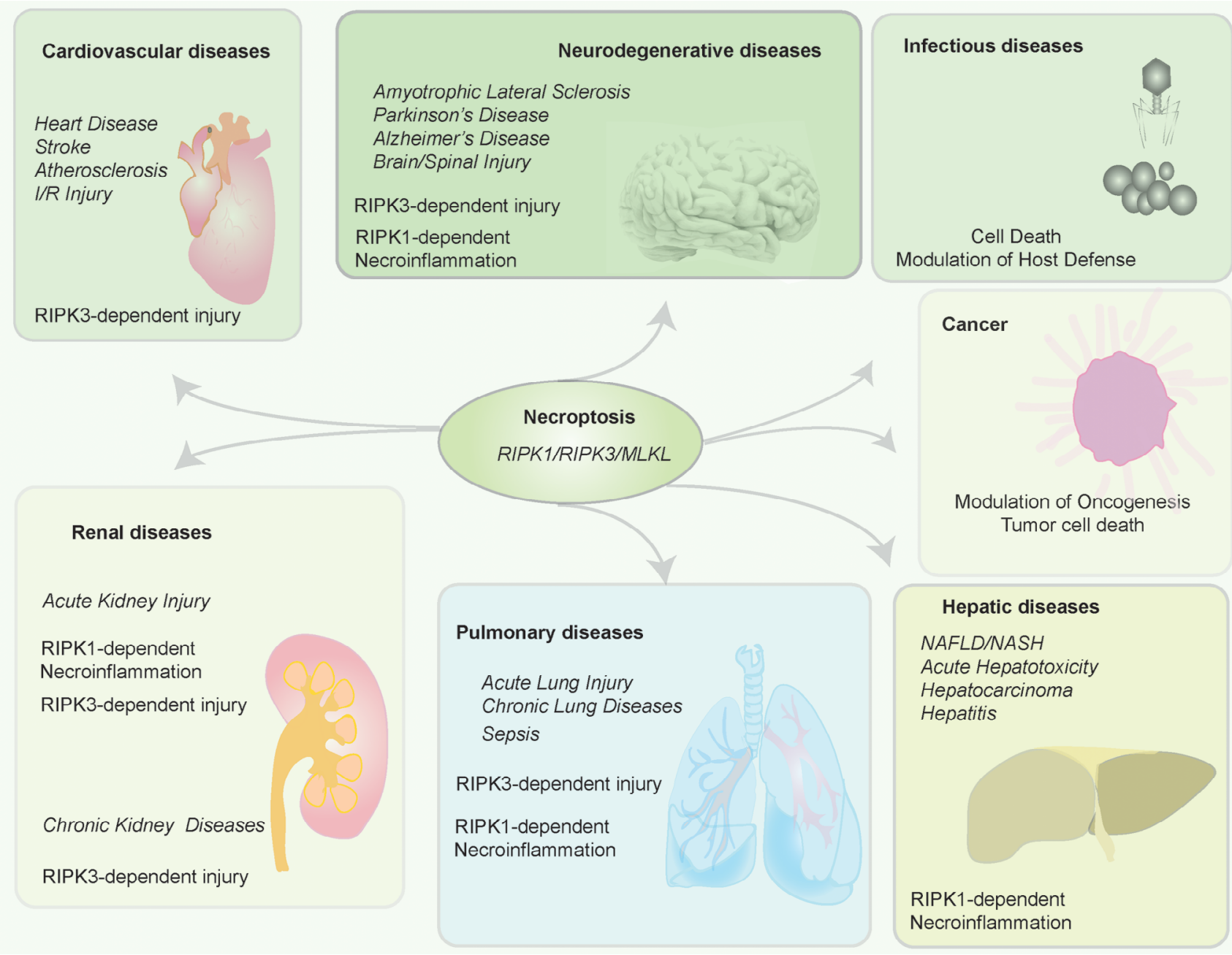

Figure 3. Central role of necroptosis in human disease. The necroptosis pathway and its regulator proteins (RIPK1, RIPK3, MLKL) have been implicated in various human diseases, including cardiovascular, neurodegenerative, infectious, hepatic, pulmonary, and renal diseases and cancer. Necroptotic cell death mediated by RIPK1/RIPK3 and/or MLKL may play a role in human diseases. In the context of infectious disease, necroptotic cell death may have a beneficial role in removing infected cells. In addition, RIPK3-dependent, but MLKL-independent, phenotypes have been observed in diseases such as acute lung and kidney injury. RIPK1-dependent necroinflammation, independent of necroptosis, has been implicated in neurodegenerative, hepatic, and renal diseases and sepsis. MLKL, mixed-lineage kinase domain-like pseudokinase; NAFLD, nonalcoholic fatty liver disease; NASH, nonalcoholic steatohepatitis; RIPK1, receptor-interacting protein kinase 1; RIPK3, receptor-interacting protein kinase 3.

ulated mitochondrial fission and activation of PINK1-dependent mitophagy, a selective autophagy subtype for the turnover of dysfunctional mitochondria (78). Genetic deficiencies in core autophagy proteins, such as microtubule-associated protein 1 light chain 3B, or mitophagy proteins, such as PINK1, were associated with epithelial cell protection from apoptotic and necrotic cell death and conferred protection against emphysema and airway dysfunction in vivo $(78,92,93)$. These studies suggest that CS-induced autophagy and mitophagy exert pro-pathogenic functions in adverse CS-induced phenotypes in mice.

We recently uncovered contributory roles for necroptosis and its regulatory proteins in chronic lung disease models (78). The RIPK1-specific inhibitor necrostatin-1 inhibited CS-induced epithelial cell death in chronic disease (78) and inhibited neutrophilic airway inflammation in CS-exposed mice (95). Genetic studies revealed that RIPK3 expression in response to CS exposure is PINK1 dependent, suggesting a role for mitochondria in necroptosis activation in response to CS (78). Thus, CS may compromise mitochondrial function and induce both mitophagy and necroptosis in pulmonary epithelium (78). In human COPD lung samples, pulmonary epithelial cells displayed increased expression of RIPK 3 and PINK1, implicating these proteins as tissue markers of CS exposure-induced emphysema and suggesting this pathway as a COPD therapeutic target $(78,81)$. 
Idiopathic pulmonary fibrosis (IPF) is a chronic, progressive lung disease characterized by progressive lung scarring (96). To date, few studies have examined the role of necroptosis in IPF. RIPK3 expression was increased in human IPF lungs, and both apoptosis and necroptosis were detected mainly in alveolar epithelial cells (AECs). In a murine bleomycin-induced (BLM-induced) pulmonary fibrosis model, RIPK3 expression was increased in AECs. Ripk3 deficiency and necrostatin-1 reduced lung inflammation and DAMP (i.e., HMGB1, IL-1 $\beta$ ) release in response to BLM. Thus, necroptosis activation in AECs and release of necrotic DAMPs may participate in pulmonary fibrogenesis (97).

\section{Necroptosis-associated proteins in renal disease}

$A K I$. Necroptosis has been implicated in the pathophysiology of AKI. In an I/R-induced AKI model, activation of RIPK3/MLKL-dependent necroptosis was observed in renal proximal tubular epithelial cells (98). Ripk3 deficiency or kinase-deficient RIPK1 prevented I/R-dependent renal tubular cell death, inflammatory cell influx, and long-term development of fibrosis (98). NLRP3 inflammasome activation and IL-1 $\beta$ secretion were also reduced in RIPK3- or MLKL-deficient mice in response to I/R. These studies proposed a "necroinflammatory" cycle that involves reciprocal stimulation of necroptosis and NLRP3 inflammasome activation and possibly underlies the transition from AKI to chronic kidney disease (CKD) (98).

Conversely, earlier studies of kidney I/R reported protection in RIPK3-deficient mice and in RIPK3 and caspase- 8 double-mutant mice, with minimal protection observed in MLKL-deficient mice (99). In a kidney transplantation model, Ripk3 deficiency in donor kidneys reduced transplant-associated I/R injury and necrosis and improved graft survival (100).

In a folic acid-induced AKI model, elevation of RIPK3 and MLKL were observed in kidney tissue; however, RIPK3 or MLKL deletion did not confer protection against renal injury at early time points (30). A ferroptosis inhibitor, but not RIPK1 inhibitor necrostatin-1, reduced injury in this model (30). During late stages of AKI, a late window of necroptosis-necrotic cell death occurred and was dependent on the TWEAK/Fn4 axis, which is known to mediate kidney injury. This study reported Ripk3 or Mlkl deficiency or necrostatin-1-mediated RIPK1 inhibition reduced late injury (101). Thus, necroptosis-dependent inflammation may exacerbate AKI at the late stage (101).

We demonstrated a crucial role for RIPK3 in the pathogenesis of sepsis-induced AKI (79). In a model of sepsis-induced AKI, using mice subjected to CLP, we demonstrated RIPK3 is upregulated in tubular epithelial cells (79). RIPK3-deficient, but not MLKL-deficient, mice were resistant to kidney tubular epithelial injury in response to CLP. RIPK3 deletion-mediated protection was associated with reduced tissue indicators of oxidative stress, including nitrotyrosine, and expression of mitochondrial NOX4. Consistently, NOX4-deficient mice were also protected in the sepsis-induced AKI model (79). Thus, RIPK3 may promote kidney tubular injury via mitochondrial dysfunction, independently of MLKL, and may represent a promising therapeutic target in sepsis-induced AKI (79).

Kidney fibrosis. Renal fibrosis is a common pathological response to injury in CKD, and RIPK3 was found to be highly expressed in human CKD (102). In 2 murine models of progressive kidney fibrosis - unilateral ureteral obstruction (UUO) or adenine diet (AD) - RIPK3 was increased in kidney tubular epithelium. Moreover, in UUO and AD models, kidney fibrosis was decreased and kidney function was improved in RIPK3-deficient mice compared with WT mice. In contrast, MLKL-deficient mice were not protected from UUO-induced kidney fibrosis. Thus, these studies have identified a novel pathway by which RIPK3 promotes kidney fibrosis independently of MLKL (102). Specifically, RIPK3 promoted fibrogenesis by stimulating AKT, resulting in downstream activation of metabolic enzyme ATP citrate lyase (ACL). Pharmacological inhibition of ACL confirmed a role for RIPK3-dependent ACL expression in UUO-mediated kidney fibrogenesis (102). These studies implicate RIPK3 in metabolic regulation and suggest RIPK3 as a promising therapeutic target in $\mathrm{CKD}$.

\section{Necroptosis-associated proteins in hepatic disease}

Necroptosis has been implicated in chronic hepatic cell death and as a modulator of major hepatic diseases, including nonalcoholic fatty liver disease (NAFLD) and nonalcoholic steatohepatitis (NASH). RIPK1-mediated inflammatory responses are important in chronic inflammation and hepatocarcinogenesis (103).

Hepatocyte injury and death, inflammation, and oxidative stress are major pathogenic features of NAFLD. Circulating markers of necrosis, TNF- $\alpha$, hepatic RIPK3, and p-MLKL were increased in human NAFLD. In high-fat, choline-deficient diet-fed or methionine- and choline-deficient (MCD) diet-fed mice, 
which serve as NASH models, RIPK3, MLKL, and TNF- $\alpha$ expression were increased in the liver. Ripk3 deficiency attenuated MCD diet-induced liver injury, steatosis, inflammation, fibrosis, and oxidative stress. Thus, RIPK3 has been implicated as a therapeutic target of NAFLD progression (104). Moreover, in patients with end-stage alcoholic cirrhosis, hepatic RIPK3 expression highly correlated with poor prognosis (105).

Mice with liver parenchymal cell-specific deletion of Ripk1 were found to be susceptible to fulminant viral hepatitis (106). Furthermore, RIPK1 deficiency exacerbated immune-dependent liver injury in mice (107). RIPK1-dependent inflammation was identified as a pathological mediator of acetaminophen-induced hepatotoxicity, excluding a role for necroptotic cell death (108). Inhibition of either RIPK1 or RIPK3 was associated with lower NLRP3 inflammasome activation in acetaminophen-induced hepatotoxicity (109). In concanavalin A-induced autoimmune hepatitis, Ripk3 deletion was protective (109), whereas RIPK1 inhibition exacerbated disease, with increased hepatocyte apoptosis and accelerated time to death. MLKL was increased in human autoimmune hepatitis and in a murine model of inflammation-dependent hepatitis. Hepatocellular necrosis in experimental hepatitis was mediated by a RIPK3-independent MLKL pathway (110). Together, these studies implicate variable roles for necroptosis-associated proteins in hepatic inflammation and disease.

\section{Necroptosis and cardiovascular disease}

Necroptosis-associated proteins have been implicated as therapeutic targets in various cardiovascular diseases, including heart failure, myocardial injury, aortic aneurysm, ischemic neural injury, and stroke (7, 111-113). In a mouse model of atherosclerosis (LDL receptor deficiency), Ripk3 and Ldlr double-knockout mice displayed reduced atherosclerotic lesions during the late state of disease progression and evidence of reduced macrophage necrosis but not apoptosis (114). Moreover, deletion of Ripk3 in apolipoprotein E-deficient mice reduced lymphocytic infiltrations, blood monocyte counts, and mortality relative to control mice $(114,115)$. RIPK3-deficient mice were also protected from adverse cardiac remodeling following I/R injury-induced myocardial infarction (116). In an elastase-induced mouse model of abdominal aortic aneurysm, Ripk3 deficiency reduced aneurism formation and inhibited TNF-mediated responses in cultured smooth muscle cells (117).

There is evidence of necroptosis activation and enhanced RIPK3 expression in hippocampal neurons in response to oxygen/glucose deprivation and in mice subjected to focal cerebral ischemia (118). In the middle cerebral artery occlusion (MCAO) model, therapeutic application of RIPK3 inhibitor GSK'872 or the pan-caspase inhibitor Z-VAD-FMK protected against ischemic brain injury. Furthermore, both GSK'872 and Ripk3 deficiency downregulated hypoxia-associated HIF-1 $\alpha$ activation and cell death (119). The RIPK3 inhibitor dabrafenib protected against focal ischemic brain injury in mice (120). RIPK1 was associated with neuroinflammation and neural cell death in rodent models of MCAO-induced stroke (121123). The RIPK1 inhibitors necrostatin-1 and 5-(3',5'-dimethoxybenzal)-2-thio-imidazole-4-ketone (DTIO) conferred protection in models of stroke by limiting neuronal injury and death (120-124).

\section{Necroptosis in neurodegenerative disease}

The necroptosis pathway and associated necroinflammation have been implicated, by pharmacological, genetic, and histological approaches, in neurodegenerative and CNS diseases, including multiple sclerosis (MS), amyotrophic lateral sclerosis (ALS), Parkinson's and Alzheimer's disease, spinal cord injury, and traumatic brain injury (TBI) (13).

MS is characterized by the loss of oligodendrocytes and demyelination. In human MS cortical lesions, defective caspase-8 activation in conjunction with RIPK1, RIPK3, and MLKL activation has been observed, accompanied by accumulation of insoluble protein aggregates. Necroptosis mediates TNF- $\alpha-$ dependent oligodendrocyte degeneration. RIPK1 inhibition protects against oligodendrocyte cell death in MS animal models, suggesting that necroptosis may be relevant to MS and that RIPK1 may represent a therapeutic target (125).

In a genetic model of Niemann-Pick disease ( $\mathrm{Npc}^{-{ }^{--}}$mice), RIPK1-specific inhibitor GSK'547 or transgenic overexpression of kinase-deficient RIPK1, but not Ripk3 deficiency, improved lifespan (126). These studies affirmed a RIPK3-independent role for RIPK1-dependent necroinflammation in disease pathogenesis (126).

Ripk3 deficiency also markedly improves neurological and systemic disease in a mouse model of the neurodegenerative disorder Gaucher's disease (GD). RIPK3-deficient GD mice had an improved clinical course, with increased survival and motor coordination, and RIPK3 deficiency exhibited protective effects on cerebral and hepatic injury (127). 
In mouse ALS models, increased activation of the RIPK3/RIPK1/MLKL-dependent necroptosis pathway was observed in neural tissue. Demyelination in the spinal cords of optineurin-deficient $\left(\mathrm{Optn}^{-/-}\right)$ ALS mice was blocked in both RIPK1- and RIPK3-deficient mice or by necrostatin-1-mediated inhibition of RIPK1. Optn ${ }^{-/-}$mice have impaired spontaneous motor activity, which was blocked by necrostatin-1 and ameliorated in RIPK1- or RIPK3-deficient mice (128). RIPK3 deficiency and inhibition of RIPK1 by Nec-1s also delayed onset of motor dysfunction in the SOD1 ${ }^{\mathrm{G} 93 \mathrm{~A}}$ mouse model of ALS (128). These studies implicate both necroptosis and RIPK1-dependent inflammation in ALS pathogenesis. In models of Parkinson's disease, pharmacological targeting of RIPK1 reduced mitochondrial morphological alterations and mitochondrial dysfunction in dopaminergic neurons (129). In models of CNS injury, such as TBI and spinal cord injury, histological studies have implicated necroptosis (130). RIPK3 deficiency protects against glial cell activation and NLRP3 inflammasome-dependent inflammation in TBI (131).

\section{Necroptosis in cancer}

A hallmark of cancer cells is resistance to apoptosis induced by anticancer agents. In cancer, necroptosis has immunogenic properties and activates antitumor responses and thereby may provide a therapeutic strategy for eliminating apoptosis-resistant tumor cells $(132,133)$. Regulation of necroptosis-associated proteins may be downregulated in certain types of tumors, including breast, colorectal, melanoma, and leukemia, whereas upregulation of RIPK1 has been reported in certain tumors (e.g., lung and pancreatic cancers) (133). Current studies also suggest pro-metastatic and immunosuppressive effects of necroptosis, which may favor cancer progression. Thus, the role of necroptosis in oncogenesis and cancer therapeutics remains incompletely delineated, can be tumor-type specific, and requires further investigation before targeting this pathway can be realized in human cancer therapies $(132,133)$.

\section{RIPK3 and related DAMPs: markers of critical illness and disease}

RIPK3 can be detected in human plasma derived from subjects in various pathophysiological states (79, $83,134,135)$. These studies investigated the significance of circulating RIPK3, as a mediator of injury and a potential marker of morbidity and mortality, in human critical illnesses, such as AKI, sepsis, and acute respiratory distress syndrome (ARDS). In critically ill patients requiring ventilator support, VILI associates with substantial morbidity and mortality. We found that plasma RIPK3 levels were higher in patients undergoing MV, compared with nonventilated intensive care unit (ICU) patients (83). In sepsis-free patients, circulating RIPK3 was higher in MV-subjected patients than those without MV, whereas no differences in plasma MLKL levels were detected. Plasma RIPK3 levels were also higher in patients with ARDS than those without ARDS. Furthermore, MV was an independent predictor of elevated plasma RIPK3 in critically ill patients (83). Given that critically ill patients may incur lipid dysregulation and metabolic dysfunction, we measured carnitine palmitoyl transferase 1 activity (CPT1), a mitochondrial enzyme governing FAO. CPT1 activity was impaired in critically ill patients compared with corresponding ICU patient controls. Impaired CPT1 activity also associated with increased plasma RIPK3 levels in critically ill patients. Collectively, our studies suggest that RIPK3, but not MLKL, is elevated in plasma of critically ill patients who undergo MV and are susceptible to VILI (83). Thus, VILI may be associated with deregulated FAO in humans and RIPK3 may mediate this association (83).

The potential of extracellular RIPK3 as a marker of human critical illness was further studied in 5 independent cohorts of critically ill patients. Plasma RIPK3 levels were higher in hospitalized patients who died compared with those who survived. Elevated plasma RIPK3 levels in patients admitted to the ICU associated with both in-hospital mortality and organ failure (134). RIPK3 levels also associated with mortality in severe sepsis cases and increased in association with blood transfusion in septic patients (135). Sepsis causes AKI in critically ill patients, although the pathophysiology remains unclear. Higher levels of RIPK3 were detected in plasma and urine in a cohort of sepsis-induced patients with AKI relative to patients without AKI (79). RIPK3 levels also increased in the systemic circulation of patients with trauma-induced AKI and correlated with AKI stage and incidence of blood transfusion (136).

Circulating mtDNA may originate from cells and tissues as the result of active necrosis or necroptosis. We have demonstrated that mtDNA levels in plasma correlated with mortality in critically ill patients with ARDS and sepsis (62). Plasma mtDNA levels in critically ill patients also correlated with altered metabolic profiles (137). Higher levels of mtDNA were also observed in both plasma and urine of sepsis-induced patients with AKI and coincided with observed elevations of RIPK3 (79). 
The occurrence of RIPK3 in the circulation may signify that necroptosis has been elevated in systemic tissues or inflammatory cells, leading to increased lytic cell death in one compartment. However, the source of plasma RIPK3 protein and other DAMPs, such as mtDNA, is difficult to ascertain. The increased levels of circulating RIPK3 may serve as a marker of inflammatory disease, which correlates with mortality in critically ill patients. Further analysis of RIPK3 in conjunction with other circulating mortality-associated markers, such as mtDNA and metabolites, may elucidate the predictive value of these markers.

\section{Conclusions and opportunities for therapy}

Collectively, recent studies have revealed a complex role for necroptosis, necroptosis pathway proteins, and necroinflammation initiated by DAMP release in orchestrating tissue injury. Cross-regulation between necroptosis and other modes of cell death increase the complexity of these pathways. The major necroptosis-regulating proteins exert pleiotropic signaling functions that culminate in necroptotic cell death and have cell death-independent functions, such as regulation of inflammasome activation, mitochondrial function and integrity, and cellular metabolic activities. RIPK3-associated injury phenotypes are not necessarily correlated with a high degree of necrotic or apoptotic cell death in tissues. Genetic deletion studies have also revealed MLKL-independent or cell death-independent roles of RIPK3 in orchestrating tissue injury and regulating immune responses $(21,83,102)$.

Targeting the necroptosis pathway may represent a therapeutic opportunity in diverse diseases, and several inhibitors of the necroptosis pathway have been developed for use in experimental settings. These inhibitors include RIPK1-specific necrostatins, RIPK3-targeting molecules (GSK'872, GSK'843, dabrafenib), the dual-specific RIPK1/RIPK3 inhibitor GSK'074, and necrosulfonamide, which targets MLKL (138-141). These small-molecule activators or inhibitors of necroptosis may be useful as therapeutics in a disease-specific manner; however, these approaches await further development and clinical testing in humans.

\section{Acknowledgments}

This work was supported in part by NIH grants R01 HL132198, R01 HL133801, R01 HL055330 to MEC and AMKC; and NIH UL1 TR000457 to DRP.

Address correspondence to: Augustine M. K. Choi, 1300 York Avenue, Suite F-113 Box 83, New York, New York 10065, USA. Phone: 212.746.6005; Email: amc2056@med.cornell.edu. Or to: Mary E. Choi, 525 East 68th Street, Box 3, New York, New York 10065, USA. Phone: 646.962.2605; Email: mechoi@med.cornell.edu.

1. Galluzzi L, et al. Molecular mechanisms of cell death: recommendations of the Nomenclature Committee on Cell Death 2018. Cell Death Differ. 2018;25(3):486-541.

2. Galluzzi L, et al. Cell death modalities: classification and pathophysiological implications. Cell Death Differ. 2007;14(7):1237-1243.

3. Majno G, Joris I. Apoptosis, oncosis, and necrosis. An overview of cell death. Am J Pathol. 1995;146(1):3-15.

4. Kroemer G, Dallaporta B, Resche-Rigon M. The mitochondrial death/life regulator in apoptosis and necrosis. Annu Rev Physiol. 1998;60:619-642.

5. Formigli L, et al. Aponecrosis: morphological and biochemical exploration of a syncretic process of cell death sharing apoptosis and necrosis. J Cell Physiol. 2000;182(1):41-49.

6. He S, Wang X. RIP kinases as modulators of inflammation and immunity. Nat Immunol. 2018;19(9):912-922.

7. Galluzzi L, Kepp O, Chan FK, Kroemer G. Necroptosis: mechanisms and relevance to disease. Annu Rev Pathol. 2017;12:103-130.

8. Weinlich R, Oberst A, Beere HM, Green DR. Necroptosis in development, inflammation and disease. Nat Rev Mol Cell Biol. 2017;18(2):127-136

9. Linkermann A, Green DR. Necroptosis. N Engl J Med. 2014;370(5):455-465.

10. Kaczmarek A, Vandenabeele P, Krysko DV. Necroptosis: the release of damage-associated molecular patterns and its physiological relevance. Immunity. 2013;38(2):209-223.

11. Tonnus W, et al. The pathological features of regulated necrosis. J Pathol. 2019;247(5):697-707.

12. Orozco S, Oberst A. RIPK3 in cell death and inflammation: the good, the bad, and the ugly. Immunol Rev. 2017;277(1):102-112.

13. Yuan J, Amin P, Ofengeim D. Necroptosis and RIPK1-mediated neuroinflammation in CNS diseases. Nat Rev Neurosci. 2019;20(1):19-33.

14. Krakauer T. Inflammasomes, autophagy, and cell death: the trinity of innate host defense against intracellular bacteria. Mediators Inflamm. 2019;2019:2471215.

15. Alvarez-Diaz S, et al. The Pseudokinase MLKL and the Kinase RIPK3 have distinct roles in autoimmune disease caused by loss 
of death-receptor-induced apoptosis. Immunity. 2016;45(3):513-526

16. Zhang X, et al. MLKL and FADD are critical for suppressing progressive lymphoproliferative disease and activating the NLRP3 inflammasome. Cell Rep. 2016;16(12):3247-3259.

17. Morgan JE, et al. Necroptosis mediates myofibre death in dystrophin-deficient mice. Nat Commun. 2018;9(1):3655.

18. Vitner EB, et al. RIPK3 as a potential therapeutic target for Gaucher's disease. Nat Med. 2014;20(2):204-208.

19. Newton K. RIPK1 and RIPK3: critical regulators of inflammation and cell death. Trends Cell Biol. 2015;25(6):347-353.

20. Newton K, Manning G. Necroptosis and inflammation. Annu Rev Biochem. 2016;85:743-763.

21. Daniels BP, et al. RIPK3 restricts viral pathogenesis via cell death-independent neuroinflammation. Cell. 2017;169(2):301-313.e11.

22. Tenev T, et al. The Ripoptosome, a signaling platform that assembles in response to genotoxic stress and loss of IAPs. Mol Cell. 2011;43(3):432-448.

23. Feoktistova M, et al. cIAPs block Ripoptosome formation, a RIP1/caspase-8 containing intracellular cell death complex differentially regulated by cFLIP isoforms. Mol Cell. 2011;43(3):449-463.

24. Lin Y, Devin A, Rodriguez Y, Liu ZG. Cleavage of the death domain kinase RIP by caspase-8 prompts TNF-induced apoptosis. Genes Dev. 1999;13(19):2514-2526.

25. Feng S, et al. Cleavage of RIP3 inactivates its caspase-independent apoptosis pathway by removal of kinase domain. Cell Signal. 2007;19(10):2056-2067.

26. Kaiser WJ, et al. RIP3 mediates the embryonic lethality of caspase-8-deficient mice. Nature. 2011;471(7338):368-372.

27. Oberst A, et al. Catalytic activity of the caspase-8-FLIP(L) complex inhibits RIPK3-dependent necrosis. Nature. 2011;471(7338):363-367.

28. Xie Y, et al. Ferroptosis: process and function. Cell Death Differ. 2016;23(3):369-379.

29. Müller T, et al. Necroptosis and ferroptosis are alternative cell death pathways that operate in acute kidney failure. Cell Mol Life Sci. 2017;74(19):3631-3645.

30. Martin-Sanchez D, et al. Ferroptosis, but not necroptosis, is important in nephrotoxic folic acid-induced AKI. J Am Soc Nephrol. 2017;28(1):218-229

31. Cheng KT, et al. Caspase-11-mediated endothelial pyroptosis underlies endotoxemia-induced lung injury. J Clin Invest. 2017;127(11):4124-4135.

32. Frank D, Vince JE. Pyroptosis versus necroptosis: similarities, differences, and crosstalk. Cell Death Differ. 2019;26(1):99-114

33. Kaczmarek A, Vandenabeele P, Krysko DV. Necroptosis: the release of damage-associated molecular patterns and its physiological relevance. Immunity. 2013;38(2):209-223.

34. Kovacs SB, Miao EA. Gasdermins: effectors of pyroptosis. Trends Cell Biol. 2017;27(9):673-684.

35. Pasparakis M, Vandenabeele P. Necroptosis and its role in inflammation. Nature. 2015;517(7534):311-320.

36. Holler N, et al. Fas triggers an alternative, caspase-8-independent cell death pathway using the kinase RIP as effector molecule. Nat Immunol. 2000;1(6):489-495.

37. Sun X, Yin J, Starovasnik MA, Fairbrother WJ, Dixit VM. Identification of a novel homotypic interaction motif required for the phosphorylation of receptor-interacting protein (RIP) by RIP3. J Biol Chem. 2002;277(11):9505-9511.

38. Sun X, Lee J, Navas T, Baldwin DT, Stewart TA, Dixit VM. RIP3, a novel apoptosis-inducing kinase. J Biol Chem 1999;274(24):16871-16875.

39. Zhang DW, et al. RIP3, an energy metabolism regulator that switches TNF-induced cell death from apoptosis to necrosis. Science. 2009;325(5938):332-336.

40. He S, et al. Receptor interacting protein kinase-3 determines cellular necrotic response to TNF- $\alpha$. Cell. 2009;137(6):1100-1111

41. Cho YS, et al. Phosphorylation-driven assembly of the RIP1-RIP3 complex regulates programmed necrosis and virus-induced inflammation. Cell. 2009;137(6):1112-1123.

42. Degterev A, et al. Identification of RIP1 kinase as a specific cellular target of necrostatins. Nat Chem Biol. 2008;4(5):313-321.

43. Newton K, Sun X, Dixit VM. Kinase RIP3 is dispensable for normal NF-kappa Bs, signaling by the B-cell and T-cell receptors, tumor necrosis factor receptor 1, and Toll-like receptors 2 and 4. Mol Cell Biol. 2004;24(4):1464-1469.

44. Kelliher MA, Grimm S, Ishida Y, Kuo F, Stanger BZ, Leder P. The death domain kinase RIP mediates the TNF-induced NF- $\kappa B$ signal. Immunity. 1998;8(3):297-303.

45. Newton K, et al. Activity of protein kinase RIPK3 determines whether cells die by necroptosis or apoptosis. Science. 2014;343(6177):1357-1360.

46. Duprez L, et al. RIP kinase-dependent necrosis drives lethal systemic inflammatory response syndrome. Immunity. 2011;35(6):908-918.

47. Polykratis A, et al. Cutting edge: RIPK1 kinase inactive mice are viable and protected from TNF-induced necroptosis in vivo. J Immunol. 2014;193(4):1539-1543.

48. Robinson N, McComb S, Mulligan R, Dudani R, Krishnan L, Sad S. Type I interferon induces necroptosis in macrophages during infection with Salmonella enterica serovar Typhimurium. Nat Immunol. 2012;13(10):954-962.

49. Kaiser WJ, et al. Toll-like receptor 3-mediated necrosis via TRIF, RIP3, and MLKL. J Biol Chem. 2013;288(43):31268-31279.

50. Takaoka A, et al. DAI (DLM-1/ZBP1) is a cytosolic DNA sensor and an activator of innate immune response. Nature. 2007;448(7152):501-505.

51. Mompeán M, et al. The structure of the necrosome RIPK1-RIPK3 core, a human hetero-amyloid signaling complex. Cell. 2018;173(5):1244-1253.e10.

52. Li J, et al. The RIP1/RIP3 necrosome forms a functional amyloid signaling complex required for programmed necrosis. Cell. 2012;150(2):339-350

53. Cai Z, et al. Plasma membrane translocation of trimerized MLKL protein is required for TNF-induced necroptosis. Nat Cell Biol. 2014;16(1):55-65.

54. Hildebrand JM, et al. Activation of the pseudokinase MLKL unleashes the four-helix bundle domain to induce membrane localization and necroptotic cell death. Proc Natl Acad Sci U S A. 2014;111(42):15072-15077.

55. Dondelinger Y, et al. MLKL compromises plasma membrane integrity by binding to phosphatidylinositol phosphates. Cell Rep 2014;7(4):971-981. 
56. Gong YN, et al. ESCRT-III acts downstream of MLKL to regulate necroptotic cell death and its consequences. Cell. 2017;169(2):286-300.e16.

57. Yoon S, Kovalenko A, Bogdanov K, Wallach D. MLKL, the protein that mediates necroptosis, also regulates endosomal trafficking and extracellular vesicle generation. Immunity. 2017;47(1):51-65.e7.

58. Li X, et al. O-GlcNAc Transferase suppresses inflammation and necroptosis by targeting receptor-interacting serine/threonine-protein kinase 3. Immunity. 2019;50(3):576-590.e6.

59. Sarhan M, Land WG, Tonnus W, Hugo CP, Linkermann A. Origin and consequences of necroinflammation. Physiol Rev. 2018;98(2):727-780.

60. Silke J, Rickard JA, Gerlic M. The diverse role of RIP kinases in necroptosis and inflammation. Nat Immunol. 2015;16(7):689-697.

61. Nakahira K, et al. Autophagy proteins regulate innate immune responses by inhibiting the release of mitochondrial DNA mediated by the NALP3 inflammasome. Nat Immunol. 2011;12(3):222-230.

62. Nakahira K, et al. Circulating mitochondrial DNA in patients in the ICU as a marker of mortality: derivation and validation. PLoS Med. 2013;10(12):e1001577; discussion e1001577.

63. Liu ZG, Hsu H, Goeddel DV, Karin M. Dissection of TNF receptor 1 effector functions: JNK activation is not linked to apoptosis while NF-kB activation prevents cell death. Cell. 1996;87(3):565-576.

64. Hsu H, Huang J, Shu HB, Baichwal V, Goeddel DV. TNF-dependent recruitment of the protein kinase RIP to the TNF receptor-1 signaling complex. Immunity. 1996;4(4):387-396.

65. Najjar M, et al. RIPK1 and RIPK3 kinases promote cell-death-independent inflammation by toll-like receptor 4. Immunity. 2016;45(1):46-59.

66. Schroder K, Tschopp J. The inflammasomes. Cell. 2010;140(6):821-832.

67. Lawlor KE, et al. RIPK3 promotes cell death and NLRP3 inflammasome activation in the absence of MLKL. Nat Commun. $2015 ; 6: 6282$.

68. Conos SA, et al. Active MLKL triggers the NLRP3 inflammasome in a cell-intrinsic manner. Proc Natl Acad Sci U S A. 2017;114(6):E961-E969.

69. Kang TB, Yang SH, Toth B, Kovalenko A, Wallach D. Caspase-8 blocks kinase RIPK3-mediated activation of the NLRP3 inflammasome. Immunity. 2013;38(1):27-40.

70. Kang S, et al. Caspase-8 scaffolding function and MLKL regulate NLRP3 inflammasome activation downstream of TLR3. Nat Commun. 2015;6:7515.

71. Zhang DW, et al. RIP3, an energy metabolism regulator that switches TNF-induced cell death from apoptosis to necrosis. Science. 2009;325(5938):332-336.

72. Lin Y, et al. Tumor necrosis factor-induced nonapoptotic cell death requires receptor-interacting protein-mediated cellular reactive oxygen species accumulation. J Biol Chem. 2004;279(11):10822-10828

73. Tait SW, et al. Widespread mitochondrial depletion via mitophagy does not compromise necroptosis. Cell Rep. 2013;5(4):878-885.

74. Wang Z, Jiang H, Chen S, Du F, Wang X. The mitochondrial phosphatase PGAM5 functions at the convergence point of multiple necrotic death pathways. Cell. 2012;148(1-2):228-243.

75. Murphy JM, et al. The pseudokinase MLKL mediates necroptosis via a molecular switch mechanism. Immunity. 2013;39(3):443-453.

76. Remijsen Q, et al. Depletion of RIPK3 or MLKL blocks TNF-driven necroptosis and switches towards a delayed RIPK1 kinase-dependent apoptosis. Cell Death Dis. 2014;5:e1004.

77. Moriwaki K, et al. The mitochondrial phosphatase PGAM5 is dispensable for necroptosis but promotes inflammasome activation in macrophages. J Immunol. 2016;196(1):407-415.

78. Mizumura K, et al. Mitophagy-dependent necroptosis contributes to the pathogenesis of COPD. J Clin Invest. 2014;124(9):3987-4003.

79. Sureshbabu A, et al. RIPK3 promotes sepsis-induced acute kidney injury via mitochondrial dysfunction. JCI Insight. 2018;3(11):98411.

80. Sauler M, Bazan IS, Lee PJ. Cell death in the lung: the apoptosis-necroptosis axis. Annu Rev Physiol. 2019;81:375-402.

81. Mizumura K, Maruoka S, Gon Y, Choi AM, Hashimoto S. The role of necroptosis in pulmonary diseases. Respir Investig. 2016;54(6):407-412.

82. Moreno-Gonzalez G, Vandenabeele P, Krysko DV. Necroptosis: a novel cell death modality and its potential relevance for critical care medicine. Am J Respir Crit Care Med. 2016;194(4):415-428.

83. Siempos II, et al. RIPK3 mediates pathogenesis of experimental ventilator-induced lung injury. JCI Insight. 2018;3(9):97102.

84. Chen J, et al. RIP3 dependent NLRP3 inflammasome activation is implicated in acute lung injury in mice. J Transl Med. 2018;16(1):233.

85. Zelic M, et al. RIP kinase 1-dependent endothelial necroptosis underlies systemic inflammatory response syndrome. J Clin Invest. 2018;128(5):2064-2075.

86. Wu J, et al. Mlkl knockout mice demonstrate the indispensable role of Mlkl in necroptosis. Cell Res. 2013;23(8):994-1006.

87. Sharma A, Matsuo S, Yang WL, Wang Z, Wang P. Receptor-interacting protein kinase 3 deficiency inhibits immune cell infiltration and attenuates organ injury in sepsis. Crit Care. 2014;18(4):R142.

88. Bolognese AC, et al. Inhibition of necroptosis attenuates lung injury and improves survival in neonatal sepsis. Surgery. 2018;S0039-6060(18):30096-30095.

89. Hansen LW, et al. Deficiency of receptor-interacting protein kinase 3 (RIPK3) attenuates inflammation and organ injury in neonatal sepsis. J Pediatr Surg. 2018;53(9):1699-1705.

90. Barnes PJ, Shapiro SD, Pauwels RA. Chronic obstructive pulmonary disease: molecular and cellular mechanisms. Eur Respir J. 2003;22(4):672-688.

91. Slebos DJ, et al. Mitochondrial localization and function of heme oxygenase-1 in cigarette smoke-induced cell death. Am J Respir Cell Mol Biol. 2007;36(4):409-417.

92. Chen ZH, et al. Egr-1 regulates autophagy in cigarette smoke-induced chronic obstructive pulmonary disease. PLoS One. 2008;3(10):e3316.

93. Chen ZH, et al. Autophagy protein microtubule-associated protein 1 light chain-3B (LC3B) activates extrinsic apoptosis during 
cigarette smoke-induced emphysema. Proc Natl Acad Sci U S A. 2010;107(44):18880-18885.

94. Ito S, et al. PARK2-mediated mitophagy is involved in regulation of HBEC senescence in COPD pathogenesis. Autophagy. 2015;11(3):547-559

95. Pouwels SD, et al. Cigarette smoke-induced necroptosis and DAMP release trigger neutrophilic airway inflammation in mice. Am J Physiol Lung Cell Mol Physiol. 2016;310(4):L377-L386.

96. Martinez FJ, et al. Idiopathic pulmonary fibrosis. Nat Rev Dis Primers. 2017;3:17074.

97. Lee JM, et al. Involvement of alveolar epithelial cell necroptosis in idiopathic pulmonary fibrosis pathogenesis. Am J Respir Cell Mol Biol. 2018;59(2):215-224.

98. Chen H, et al. RIPK3-MLKL-mediated necroinflammation contributes to AKI progression to CKD. Cell Death Dis. 2018;9(9):878

99. Newton K, et al. RIPK3 deficiency or catalytically inactive RIPK1 provides greater benefit than MLKL deficiency in mouse models of inflammation and tissue injury. Cell Death Differ. 2016;23(9):1565-1576.

100. Lau A, et al. RIPK3-mediated necroptosis promotes donor kidney inflammatory injury and reduces allograft survival. $A m J$ Transplant. 2013;13(11):2805-2818.

101. Martin-Sanchez D, et al. TWEAK and RIPK1 mediate a second wave of cell death during AKI. Proc Natl Acad Sci U S A. 2018;115(16):4182-4187.

102. Imamura M, et al. RIPK3 promotes kidney fibrosis via AKT-dependent ATP citrate lyase. JCI Insight. 2018;3(3):94979.

103. Kondylis V, Pasparakis M. RIP kinases in liver cell death, inflammation and cancer. Trends Mol Med. 2019;25(1):47-63.

104. Afonso MB, et al. Necroptosis is a key pathogenic event in human and experimental murine models of non-alcoholic steatohepatitis. Clin Sci. 2015;129(8):721-739.

105. Zhang Z, et al. RIPK3-mediated necroptosis and neutrophil infiltration are associated with poor prognosis in patients with alcoholic cirrhosis. J Immunol Res. 2018;2018:1509851.

106. Farooq M, et al. Depletion of RIPK1 in hepatocytes exacerbates liver damage in fulminant viral hepatitis. Cell Death Dis 2019;10(1):12

107. Suda J, et al. Knockdown of RIPK1 markedly exacerbates murine immune-mediated liver injury through massive apoptosis of hepatocytes, independent of necroptosis and inhibition of NF-кB. J Immunol. 2016;197(8):3120-3129.

108. Dara L, et al. Receptor interacting protein kinase 1 mediates murine acetaminophen toxicity independent of the necrosome and not through necroptosis. Hepatology. 2015;62(6):1847-1857.

109. Deutsch M, et al. Divergent effects of RIP1 or RIP3 blockade in murine models of acute liver injury. Cell Death Dis. 2015;6:e1759.

110. Günther C, et al. The pseudokinase MLKL mediates programmed hepatocellular necrosis independently of RIPK3 during hep atitis. J Clin Invest. 2016;126(11):4346-4360.

111. Jun-Long H, et al. Necroptosis signaling pathways in stroke: from mechanisms to therapies. Curr Neuropharmacol. 2018;16(9):1327-1339.

112. Gupta K, Phan N, Wang Q, Liu B. Necroptosis in cardiovascular disease - a new therapeutic target. J Mol Cell Cardiol. 2018;118:26-35

113. Zhu H, Sun A. Programmed necrosis in heart disease: Molecular mechanisms and clinical implications. J Mol Cell Cardiol. 2018;116:125-134.

114. Lin J, et al. A role of RIP3-mediated macrophage necrosis in atherosclerosis development. Cell Rep. 2013;3(1):200-210.

115. Meng L, Jin W, Wang X. RIP3-mediated necrotic cell death accelerates systematic inflammation and mortality. Proc Natl Acad Sci U S A. 2015;112(35):11007-11012.

116. Luedde M, et al. RIP3, a kinase promoting necroptotic cell death, mediates adverse remodelling after myocardial infarction. Cardiovasc Res. 2014;103(2):206-216.

117. Wang Q, Liu Z, Ren J, Morgan S, Assa C, Liu B. Receptor-interacting protein kinase 3 contributes to abdominal aortic aneurysms via smooth muscle cell necrosis and inflammation. Circ Res. 2015;116(4):600-611.

118. Vieira $\mathrm{M}$, et al. Ischemic insults induce necroptotic cell death in hippocampal neurons through the up-regulation of endogenous RIP3. Neurobiol Dis. 2014;68:26-36.

119. Yang XS, et al. Hypoxia-inducible factor-1 alpha is involved in RIP-induced necroptosis caused by in vitro and in vivo ischemic brain injury. Sci Rep. 2017;7(1):5818.

120. Cruz SA, Qin Z, Stewart AFR, Chen HH. Dabrafenib, an inhibitor of RIP3 kinase-dependent necroptosis, reduces ischemic brain injury. Neural Regen Res. 2018;13(2):252-256.

121. Ni Y, et al. RIP1K contributes to neuronal and astrocytic cell death in ischemic stroke via activating autophagic-lysosomal pathway. Neuroscience. 2018;371:60-74.

122. Chen Y, et al. Necrostatin-1 improves long-term functional recovery through protecting oligodendrocyte precursor cells after transient focal cerebral ischemia in mice. Neuroscience. 2018;371:229-241.

123. Li W, et al. Neuroprotective effects of DTIO, a novel analog of Nec-1, in acute and chronic stages after ischemic stroke. Neuroscience. 2018;390:12-29.

124. Degterev A, et al. Chemical inhibitor of nonapoptotic cell death with therapeutic potential for ischemic brain injury. Nat Chem Biol. 2005;1(2):112-119.

125. Ofengeim D, et al. Activation of necroptosis in multiple sclerosis. Cell Rep. 2015;10(11):1836-1849.

126. Cougnoux A, Clifford S, Salman A, Ng SL, Bertin J, Porter FD. Necroptosis inhibition as a therapy for Niemann-Pick disease, type C1: Inhibition of RIP kinases and combination therapy with 2-hydroxypropyl- $\beta$-cyclodextrin. Mol Genet Metab. 2018;125(4):345-350.

127. Vitner EB, et al. RIPK3 as a potential therapeutic target for Gaucher's disease. Nat Med. 2014;20(2):204-208.

128. Ito Y, et al. RIPK1 mediates axonal degeneration by promoting inflammation and necroptosis in ALS. Science. 2016;353(6299):603-608.

129. Iannielli A, et al. Pharmacological inhibition of necroptosis protects from dopaminergic neuronal cell death in Parkinson's dis ease models. Cell Rep. 2018;22(8):2066-2079. 
130. Liu S, et al. Lysosomal damage after spinal cord injury causes accumulation of RIPK1 and RIPK 3 proteins and potentiation of necroptosis. Cell Death Dis. 2018;9(5):476.

131. Liu ZM, et al. RIP3 deficiency protects against traumatic brain injury (TBI) through suppressing oxidative stress, inflammation and apoptosis: Dependent on AMPK pathway. Biochem Biophys Res Commun. 2018;499(2):112-119.

132. Krysko O, et al. Necroptotic cell death in anti-cancer therapy. Immunol Rev. 2017;280(1):207-219.

133. Gong Y, et al. The role of necroptosis in cancer biology and therapy. Mol Cancer. 2019;18(1):100.

134. Ma KC, et al. Circulating RIPK3 levels are associated with mortality and organ failure during critical illness. JCI Insight. 2018;3(13):99692.

135. Qing DY, et al. Red blood cells induce necroptosis of lung endothelial cells and increase susceptibility to lung inflammation. Am J Respir Crit Care Med. 2014;190(11):1243-1254.

136. Shashaty MG, et al. Plasma levels of receptor interacting protein kinase-3 (RIP3), an essential mediator of necroptosis, are associated with acute kidney injury in critically ill trauma patients. Shock. 2016;46(2):139-143.

137. Johansson PI, et al. Plasma mitochondrial DNA and metabolomic alterations in severe critical illness. Crit Care. 2018;22(1):360.

138. Wang Z, et al. Using drugs to target necroptosis: dual roles in disease therapy. Histol Histopathol. 2018;33(8):773-789.

139. Degterev A, Ofengeim D, Yuan J. Targeting RIPK1 for the treatment of human diseases. Proc Natl Acad Sci U S A. 2019;116(20):9714-9722.

140. Cruz SA, Qin Z, Stewart AFR, Chen HH. Dabrafenib, an inhibitor of RIP3 kinase-dependent necroptosis, reduces ischemic brain injury. Neural Regen Res. 2018;13(2):252-256.

141. Zhou T, et al. Identification of a novel class of RIP1/RIP3 dual inhibitors that impede cell death and inflammation in mouse abdominal aortic aneurysm models. Cell Death Dis. 2019;10(3):226.

142. Campisi J. Aging, cellular senescence, and cancer. Annu Rev Physiol. 2013;75:685-705. 